\title{
CUANTIFICACIÓN DEL CARBONO ALMACENADO EN FORMACIONES VEGETALES AMAZÓNICAS EN “CICRA”, MADRE DE DIOS (PERÚ)
}

\section{QUANTIFICATION OF THE CARBON STORAGE IN AMAZON VEGETATION TYPES AT “CICRA”, MADRE DE DIOS (PERU)}

\author{
${\text { Carlos } \text { Martel }^{1} \text { y Lianka Cairampoma }}^{1}$
}

\begin{abstract}
Resumen
La llanura amazónica peruana se caracteriza por la presencia de múltiples formaciones vegetales. Éstas cada vez reciben mayor impacto por actividades antropogénicas tales como la minería y tala. Todo esto, sumado al cambio climático global, genera desconcierto sobre el futuro de los bosques. La identificación de los niveles de almacenamiento de carbono en áreas boscosas, y específicamente en cada formación vegetal, permitiría un mejor manejo de las zonas de conservación, así como identificar las áreas potenciales que servirían para el financiamiento de la absorción de carbono y otros servicios ambientales. El presente estudio fue desarrollado en la estación Biológica del Centro de Investigación y Capacitación Río Los Amigos (CICRA). En el CICRA se identificaron tres formaciones vegetales principales, el bosque de terraza, el bosque inundable y el aguajal. Siendo los bosques de terraza los de mayor extensión y mayor cantidad de carbono acumulado. Como resultado se valorizó la vegetación presente en el CICRA, en alrededor de 11 millones de dólares americanos. El ingreso a la oferta de los bonos de carbono promovería la conservación de los bosques.
\end{abstract}

Palabras clave: Bosques tropicales, Amazonia peruana, cambio climático global, conservación, GIS.

\begin{abstract}
The Peruvian Amazon Basin is characterized by the presence of multiple vegetation types. They are being given great impact by human activities such as mining and, logging. All this, coupled with global climate change, creates confusion about the future of our forests. The identification of levels of carbon storage in forested areas, and specifically in each vegetation type, would allow better management of conservation areas, and then identify potential areas that could serve to finance carbon sequestration and other environmental services. This study was conducted at the Biological Station for Research and Training Center Rio Los Amigos (CICRA, Spanish acronym). At the station three main formations were identified, alluvial terrace forests, flood terrace forests and Mauritia swamps. The alluvial terrace forest presents the most extensive area and the highest amount of carbon stored. As result, CICRA vegetations were valued at approx. 11 millions U.S. dollars. Admission to the supply of carbon credits could promote Amazon forest conservation.
\end{abstract}

Key words: Tropical forests, Peruvian Amazon, global climate change, conservation, GIS.

\section{Introducción.}

El aumento de la concentración de dióxido de carbono $\left(\mathrm{CO}_{2}\right)$ en la atmósfera de la Tierra es una preocupación mundial al ser considerado como uno de los 6 gases que intervienen en el calentamiento global (IPCC, 2001). La concentración de este gas en la atmósfera aumentó de 280 ppm en el año 1750, a 379 ppm en el 2004, y continúa incrementándose a una tasa anual mayor a 1 ppm (Epstein \& Rogers, 2004; NETL, 2004), debido principalmente al uso de combustibles fósiles que generan del 80 al $85 \%$ del $\mathrm{CO}_{2}$ emitido (Andrade \& Ibrahim, 2003). Algunas manifestaciones del calentamiento global implican el incremento de la temperatura en casi $0.5^{\circ} \mathrm{C}$ desde el siglo pasado (Ciesla, 1996) y cambios en los regímenes hídricos. Estos cambios pueden reducir la estabilidad y la reserva de carbono orgánico presente en el suelo, el aumento de la susceptibilidad del suelo a la escorrentía y la erosión, y alterar los ciclos del agua, carbono, nitrógeno, fósforo, azufre y otros elementos, causando efectos negativos en la productividad de biomasa, la biodiversidad y el medio ambiente (IPCC, 2001).

Las plantas utilizan $\mathrm{CO}_{2}$ y liberan $\mathrm{O}_{2}$ durante el proceso fotosintético; asimismo, almacenan componentes de carbono en sus estructuras por periodos prolongados, por lo cual se les considera como reservas naturales de carbono (Schlesinger \& 
Andrews, 2000). El tiempo desde que el carbono se encuentra constituyendo alguna estructura de la planta hasta que es enviado al suelo o la atmósfera, se considera almacenado (Rodríguez et al., 2006). Por otro lado, la capacidad de los ecosistemas para almacenar carbono en forma de biomasa aérea, varía en función de la edad, diámetro, altura de los componentes arbóreos, la densidad de la población de cada estrato y de la comunidad vegetal (Alegre et al., 2000). La capacidad de fijación de carbono por los ecosistemas forestales aún es desconocida, debido al uso de diferentes métodos que miden diferentes flujos del ciclo terrestre del carbono (Schulze et al., 2000; Díaz et al., 2007).

Los bosques son los almacenes más importantes del mundo (Jandl, 2001), y son responsables de la mayor parte de los flujos de carbono entre la tierra y la atmósfera a través de la fotosíntesis y la respiración (Tipper, 1998). Aproximadamente el 90\% de la biomasa acumulada en la tierra se encuentra en los bosques en forma de fustes, ramas, hojas, raíces y materia orgánica (Leith \& Whithacker, 1975; Raev et al., 1997). De éstos, el tipo Bosque Tropical domina claramente sobre los otros bosques del mundo (40\% de área global de bosques representados). Por ello, el rol del Bosque Tropical predomina por sobre los demás bosques en el ciclo del carbono, basado en el flujo y volumen almacenado de carbono (Butcher et al., 1998) y por tanto la gran importancia de su conservación como sumidero de carbono.

Según los trabajos realizados en Perú entre 1983 y 2007 basados en compilaciones de datos de campo (Houghton, 1999; DeFries et al., 2002; Gibbs, 2006; IPCC, 2006) e inventarios florísticos del bosque (Brown, 1992; Achard et al., 2002), calculan las concentraciones de carbono almacenado entre los 2 782 y 13241 MtC (Gibbs et al., 2007), siendo uno de los más elevados en el mundo. Esta situación resalta la importancia del estudio por formaciones vegetales (ver Iverson et al., 1993) ya que cada formación vegetal varía en la distribución de los nutrientes del suelo y la disponibilidad de agua, factores que condicionan las adaptaciones vegetales y favorecen el crecimiento de comunidades típicas (Encarnación, 1993) y por tanto la composición florística.

En muchos estudios se ha empleado la biomasa de los árboles para estimar su contenido de carbono, a través de la multiplicación de la cantidad disponible en una determinada superficie por un factor que va desde 0.40 hasta el 0.55 (Figueroa et al., 2005), ya que se ha encontrado que esta es la proporción de carbono contenido en cualquier especie vegetal (Díaz et al., 2007). Según Husch (2001), para estimar la biomasa en un sistema forestal, y por tanto el contenido de carbono, se requiere de: un inventario de árboles en pie (vivos o muertos), la cuantificación de la vegetación no arbórea, la estimación de la biomasa muerta (necromasa) y la estimación de biomasa en las raíces y el suelo.

En el contexto nacional, la extracción de madera nativa (tala), genera empleo y beneficios económicos, y la conversión del bosque a pastos o cultivos, provee de alimentos y oportunidades de inversión; sin embargo estas actividades degradan los bosques e incrementan los efectos del calentamiento global. Una alternativa de desarrollo sostenible podría ser el financiamiento de la absorción de carbono de los bosques de nuestra Amazonía. El costo por tonelada de carbono almacenado usualmente es superior a 5 USD por tonelada (Concha et al., 2007), alcanzando los 10 USD por tonelada (Kerr et al., 2004) y estimaciones previas lo valoraban en el rango de 10 a 20 USD (Kerr et al., 2004). Por ejemplo durante el 2007, el precio promedio por tonelada de carbono almacenado fue de 6.13 USD. Por ello, el financiamiento del carbono podría ser una herramienta clave en los proyectos de conservación y mitigación ambiental.

Por tanto, el objetivo de este trabajo fue: (a) estimar el carbono almacenado en las distintas formaciones vegetales presentes en los bosques del Centro de investigación y Capacitación Rio Los Amigos (CICRA), (b) identificar entre estas, las que almacenan mayores cantidades de carbono, y (c) valorizar según el precio del mercado el carbono almacenado en las mismas.

\section{Materiales y métodos.}

Área de estudio.

El proyecto se realizó en el Centro de Investigación y capacitación río Los Amigos (CICRA). Ubicado en la cuenca del río Los Amigos (12³4'8.51"S 705'57.73"W), Departamento de Madre de Dios, Perú (Figura 1). El CICRA ocupa una terraza por encima del río Madre de Dios. El bosque de terraza y el bosque inundable son los que predominan. Además, el CICRA presenta en sus alrededores parches de bambú, bosque de sucesión primaria, vegetación de ribera y zonas alteradas por la minería de oro artesanal (para más información ver Pitman, 2008). Se encuentra entre los 230 y 270 m de elevación. Presenta una temperatura y una precipitación promedio anual de $24^{\circ} \mathrm{C}$ y $2648 \mathrm{~mm}$, respectivamente (Ascorra et al., 1999).

En base a los criterios de clasificación de las formaciones vegetales de Encarnación (1993) para la llanura amazónica y el mapa de vegetación del área de Winrock (2006), se clasificaron las diferentes formaciones vegetales del CICRA. En tal sentido el criterio aplicado fue: si la vegetación se encuentra estacionalmente inundada o no, y si estas vegetaciones presentan dominancia de una o unas pocas especies. Cálculo del área ocupada por cada formación vegetal.

Se trabajó con imágenes Landsat 5 Thematic Mapper $(\mathrm{TM})($ path $=3$ y row $=69)$ adquirida el 23 de 
Agosto del 2007, correspondientes al área de estudio. El tamaño en pixeles nominales de la data fue 30 x 30 m. Para visualizar bosques y áreas descubiertas se usó la combinación de bandas 5,4,3 (R,G,B), mientras que para visualizar las áreas inundadas se usó la combinación 4,5,3 (R,G,B) (Chuvieco, 1995).

Mediante los programas ArcGis 9.3 y Envi 4.6.1 se logró discernir las distintas formaciones vegetales. Se usó la reflectancia de cada formación vegetal y la georeferenciación de los puntos de muestreo tomados en campo para la delimitación de las mismas. En tanto que, para diferenciar parches de carrizales-pacales del resto de la vegetación, se enmascaró y clasificó la imagen satelital en tres categorías (bosque, carrizalpacal y área descubierta), basándose en las diferentes reflectancias que estas tres categorías emiten. Posteriormente, con la imagen enmascarada, se logró las medidas de 701 individuos arbóreos en un total de 10 parcelas de $100 \mathrm{~m}^{2}$.

Mediante el uso de medidas alométricas se calculó la masa seca de la biomasa aérea siguiendo la ecuación propuesta por Winrock (2006), para especies amazónicas. Esta es:

Winrock (2006): Biomasa árbol $=0.22582367$ * (D) $)^{2.4049471}$

Donde:

D: Diámetro del tronco a la altura del pecho $(1.3 \mathrm{~m})$ en centímetros.

Las lianas no fueron incluidas en el inventario debido a que en muchos casos no es posible calcular su longitud, la cual que se enmascara con el dosel.

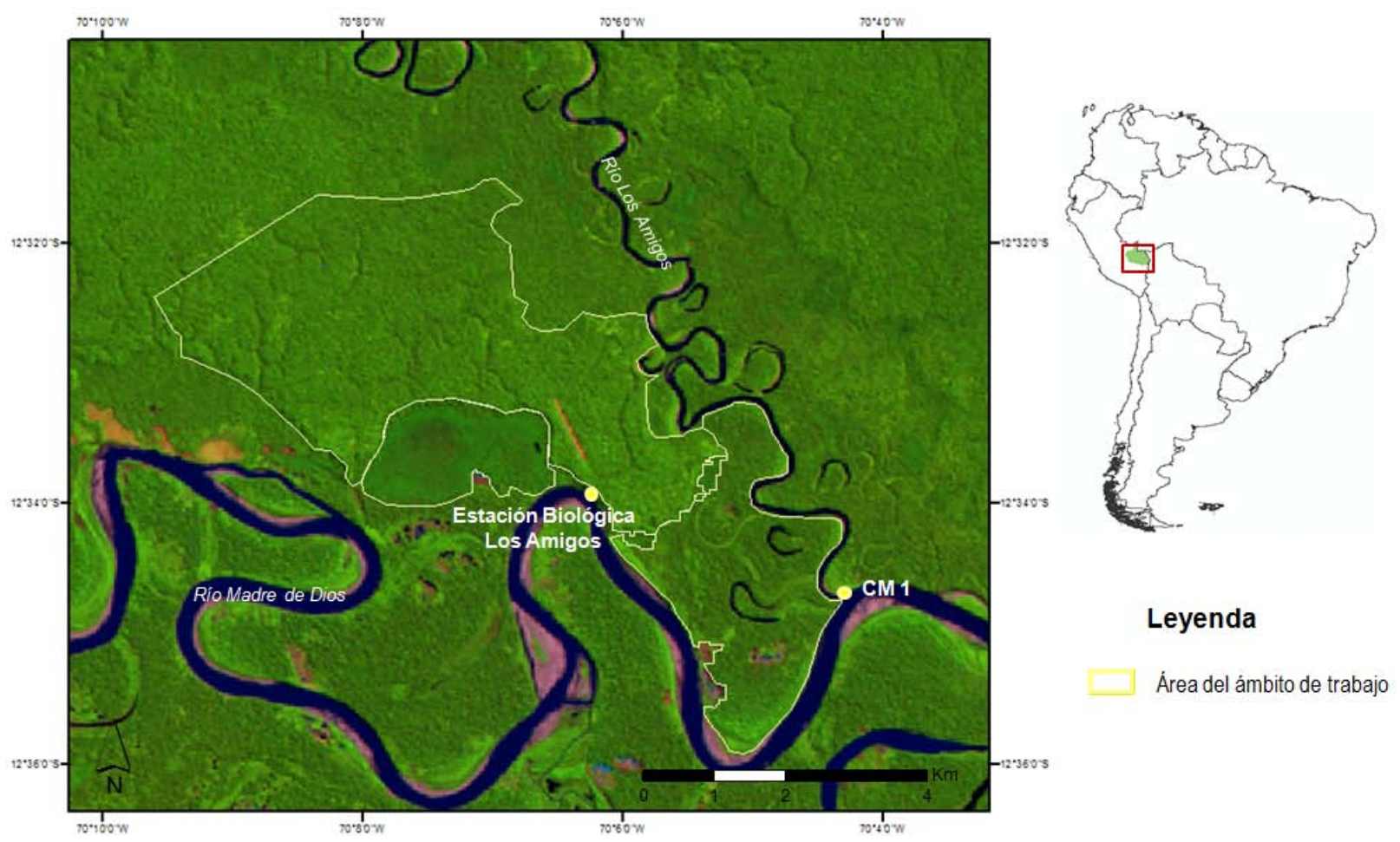

Figura 1. Mapa de ubicación del Centro de investigación y Capacitación río Los Amigos (CICRA) y ámbito de trabajo.

cuantificar las hectáreas que abarcan cada una de las categorías asignadas. Mediante el número de pixeles fue posible cuantificar el área de cada formación.

Cálculo de la biomasa aérea y del carbono almacenado por formación vegetal.

En cada formación vegetal se realizó el muestreo de: (i) biomasa arbórea, en parcelas de 4x25 m; (ii) biomasa arbustiva y herbácea, en cuadrantes de 1x1 $\mathrm{m}$, dentro de las parcelas de $4 \times 25 \mathrm{~m}$; y (iii) biomasa muerta (necromasa), incluyendo hojarasca y árboles caídos o de pie muertos, en subcuadrantes de $0.5 \times 0.5$ $\mathrm{m}$ dentro del cuadrante de $1 \mathrm{x} 1 \mathrm{~m}$. En total se tomaron
Para el cálculo del carbono almacenado se multiplicó la biomasa obtenida por 0.50, el factor promedio de carbono registrado en plantas (IPCC, 1996).

Dado que los aguajales son formaciones vegetales generalmente homogéneas, conformadas casi exclusivamente por Mauritia flexuosa, se estimó el carbono almacenado mediante los cálculos realizados por Freitas et al. (2006) en aguajales de la Reserva Nacional Pacaya Samiria.

Valoración del Carbono Almacenado.

Para la valoración del carbono se utilizó el precio de un área neotropical con similares características al CICRA. Por ello se utilizó el valor estimado promedio 
de 15 dólares americanos calculado para bosques manejados de Costa Rica (Kerr et al., 2001; Richards y Stokes, 2004).

\section{Resultados.}

Cálculo del área ocupada por cada formación vegetal.

Se identificaron cinco

formaciones vegetales: (a) bosque de terraza (o de altura), (b) bosque inundable (o bajío), (c) aguajal, (d) pacal y (e) carrizales. De estos, las tres primeras formaciones fueron las predominantes en cuanto a su extensión. La formación vegetal con mayor extensión dentro del CICRA fue el bosque de terraza con 1887.18 ha; mientras que la menor fue el de carrizalpacal con 15.12 ha (ver Figura 2). Las formaciones vegetales fueron fácilmente distinguibles mediante imágenes satelitales, excepto en el caso del carrizal y pacal. Esto debido probablemente a la similitud en la reflectancia entre estas dos formaciones vegetales, así como el solapamiento de los carrizos por el dosel arbóreo (ver Tabla 1).

Cálculo de la biomasa aérea y carbono por formación vegetal.

El bosque de terraza fue la formación vegetal con mayor cantidad de carbono almacenado con 335.11 tC $\mathrm{ha}^{-1}$, esta cifra no incluye un árbol de $500 \mathrm{~cm}$ de diámetro ya que distorsionaba mucho la cifra (854 tC $\mathrm{ha}^{-1}$ ) y árboles de este diámetro son raramente
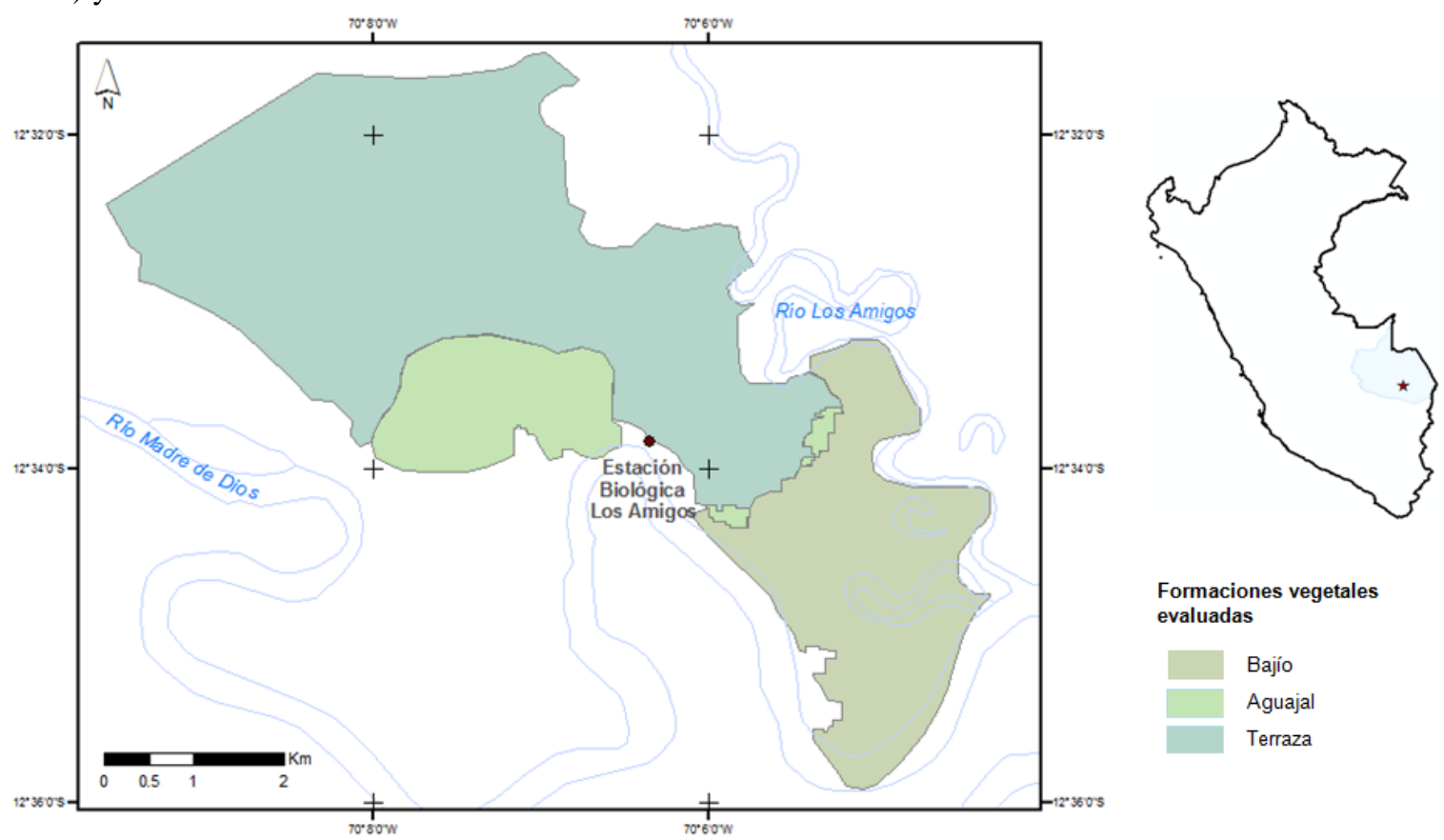
evaluadas

$$
\begin{aligned}
& \text { Bajío } \\
& \text { Aguajal } \\
& \text { Terraza }
\end{aligned}
$$
inundable con una cantidad almacenada de $141.81 \mathrm{tC}$ ha $^{-1}$. El aguajal ha sido registrado con una biomasa aérea de $115.40 \mathrm{tC} \mathrm{ha}^{-1}$ (y superior a las $400 \mathrm{tC} \mathrm{ha}^{-1} \mathrm{si}$ se incluye la parte subterránea; Freitas et al., 2006). Mientras, las formaciones con menor cantidad de carbono almacenado fueron el carrizal y el pacal con 3.55 y 39.87 tC ha $^{-1}$, respectivamente (ver Tabla 1 ).

La cantidad total de carbono almacenado dentro del área (2 977.896 ha) fue de 776603.28 tC. Siendo el promedio por hectárea de 260.79 tC. De las

Tabla 2. Comparación en la cantidad de carbono almacenado por unidad de muestreo en las dos principales formaciones vegetales.

*La necromasa incluye los troncos de árboles muertos de pie y caídos.

Tabla 1. Relación de la formación vegetal con la cantidad de carbono

\begin{tabular}{lrrr} 
Formación Vegetal & Área (ha) & $\begin{array}{c}\text { Promedio carbono } \\
\text { almacenado (tC /ha) }\end{array}$ & $\begin{array}{r}\text { Total carbono } \\
\text { almacenado } \\
\text { (tC) }\end{array}$ \\
\hline Bosque de terraza & 1887.18456 & 335.1101 & 632414.607 \\
Bosque inundable & 736.86077 & 141.8128 & 104496.289 \\
Aguajal & 338.731 & $115.4^{*}$ & 39089.5574 \\
Pacal-carrizal & 15.12 & $39.8697^{\gamma}$ & 602.8299 \\
TOTAL & 2977.8962 & 260.7892 & 776603.283 \\
\hline
\end{tabular}
Unidad de Bosque de terraza $\left(\mathrm{kg} / \mathrm{m}^{2}\right) \quad$ Bosque inundable muestreo $\left(\mathrm{kg} / \mathrm{m}^{2}\right)$

$\begin{array}{ccc}\text { Árboles } & 30.2213 & 11.5113 \\ \text { Herbáceas } & 0.8125 & 0.1813\end{array}$

Herbáceas $\quad 0.8125 \quad 0.1813$


formaciones vegetales, la que más aporta en la cantidad total de carbono almacenado es el bosque de terraza (ver Tabla 1). Resulta notable la diferencia entre la cantidad de carbono almacenado en herbáceas y necromasa en el bosque inundable $\left(0.18 \mathrm{~kg} / \mathrm{m}^{2}\right)$ y el bosque de terraza $\left(0.81 \mathrm{~kg} / \mathrm{m}^{2}\right)$, un valor superior a cuatro veces, ya que este último almacena en los árboles casi tres veces el carbono que almacena el bosque inundable (Tabla 2).

Valoración del Carbono Almacenado.

En el área de estudio el carbono que se encuentra almacenado en los bosques totaliza de 776603.28 tC. El CICRA es un área de manejo y concesión por lo que se utilizó el valor de 15 dólares americanos por tonelada de carbono almacenado (Kerr et al., 2001). El resultado fue de $11 \quad 649049.2$ (once millones seiscientos cuarenta y nueve mil cuarenta y nueve con 20/100) dólares americanos.

\section{Discusión.}

La cantidad promedio de carbono almacenado registrada aquí, $260.79 \mathrm{tC} \mathrm{ha}^{-1}$, es muy superior a los 173 tC ha $^{-1}$ registrada por Winrock (2006) a pesar de usar la misma ecuación. Esto se explica porque el área estudiada por Winrock (2006) presenta una mayor diversidad de formaciones vegetales que no se registran en CICRA. Mientras, el IPCC (2000) reportó un promedio de 270 tC ha $^{-1}$ para bosques amazónicos, ligeramente superior a lo descrito. Esto posiblemente por una diferencia en el número de individuos reportados por área muestral; y principalmente, como señalan Schulze et al., (2000), el uso de distintos métodos para la estimación de carbono. En otros reportes, Nascimento y Laurance (2002) reportan alrededor de $199 \pm 15 \mathrm{tC} \mathrm{ha}^{-1}$, en un estudio que se llevó a cabo en áreas no disturbadas, donde se espera mayor biomasa de la vegetación. Reportes anteriores para la Amazonía registran cantidades muy inferiores, entre 80 y 160 tC ha-1 (Fearnside, 1992, 1994, 1997; Brown \& Lugo, 1992; Phillips et al., 1998).

La formación vegetal con mayor cantidad de carbono almacenado fue el bosque de terraza. El bosque de terraza presenta una elevada cantidad de carbono almacenada 335.11 tC ha ${ }^{-1}$, similar a anteriores reportes. Márquez (2000) registra 410 tC ha $^{-1}$, Barbarán (1998) 300 tC ha ${ }^{-1}$, el IIAP (2002) $226.19 \mathrm{tC} \mathrm{ha}^{-1}$, y Freitas et al. (2006) $484 \mathrm{tC} \mathrm{ha}^{-1}$. Winrock (2006) reporta una menor cantidad de carbono almacenado en los bosques de terraza en comparación con los bosques inundables; señaló que una de las razones fue el menor desarrollo de la vegetación debido a los limitados nutrientes y el impacto de la tala. La vegetación más desarrollada (número de especímenes arbóreos y altura de estas) se da en el bosque de terraza. Únicamente se encontró mayor cantidad de carbono almacenado en el sustrato herbáceo del bosque inundable. Esto se explica por las periódicas inundaciones que eliminan y renuevan las hierbas.

La formación de pacal se encuentra distribuida en pequeños parches rodeados por el bosque de terraza. Siendo el cálculo del carbono almacenado mucho menor al presentado por Winrock (2006). Aunque aquí solo representó el $1 \%$ de todo el carbono almacenado en el área debido a su poca biomasa, la casi exclusiva presencia de Guadua spp. y la pequeña área en la que está presente. La biomasa registrada en la literatura para pacales en Brasil es de 240.9 t ha $^{-1}$ (Torezan \& Silveira, 2000), conteniendo alrededor de 120 tC ha $^{-1}$. Esta cifra es muy superior a lo aquí registrado; aunque se encuentra en el rango de los bosques de bambú asiáticos (aproximadamente de 15.05 $\mathrm{Ct} \mathrm{ha}^{-1}$ a $80.85 \mathrm{tC} \mathrm{ha}^{-1}$; Isagi et al., 1993).

\section{Conclusiones.}

Se identificó a los bosques de terraza como la formación vegetal que almacena mayores niveles de carbono en sus árboles, y son por tanto la formación vegetal de mayor importancia para la conservación y manejo.

Respecto de la valoración del carbono almacenado, se calculó un estimado superior a los 11 millones de dólares americanos, que representaría el $0.025 \%$ del PBI nacional anual, como posibles ingresos en mercado de carbono. Esto a su vez significaría alrededor del $8 \%$ del PBI anual para el departamento de Madre de Dios (INEI, 2010).

Cabe destacar, que esta estimación monetaria no incluye el carbono almacenado subterráneamente, el mismo que podría representar desde el $10 \%$ al $40 \%$ del carbono (o incluso hasta el 80\%) de un ecosistema, ni el flujo anual (captación) del $\mathrm{CO}_{2}$ ambiental, por lo que este valor podría verse incrementado.

El potencial de los bosques en los alrededores del CICRA, son una muestra de lo registrado en otras áreas de la Amazonía. Por lo tanto, ingresar a la oferta de carbonos sería una estrategia para promover la conservación y el manejo sostenible de esta estación biológica, y de los ecosistemas amazónicos en general.

\section{Agradecimientos.}

El desarrollo del presente trabajo no hubiera sido posible sin el apoyo económico de la Asociación para la Conservación de la Cuenca Amazónica (ACCA) e IdeaWild por el apoyo con equipos de campo.

\section{Literatura citada.}

Achard F., Eva H.D., Stibig H-J., Mayaux P., Gallego J., Richards T. \& Malingreau J-P. 2002. Determination of deforestation rates of the world's human tropical forests. Science. 297: 999-1002.

Alegre J., Ricse A., Arévalo L., Barbarán J. \& Palm C. 2000. Reservas de carbono en diferentes sistemas de uso de la tierra en la amazonía peruana. Consorcio para el Desarrollo Sostenible de Ucayali (CODESU). Boletín informativo. 12: 8-9. 
Andrade H.J. \& Ibrahim M. 2003. ¿Cómo monitorear el secuestro de carbono en los sistemas silvopastoriles? Agroforesteria en las Américas. 10: 39-40.

Ascorra C., Barreda R., Chicchon A., Chonati A. M., Dávalos L., Espinel L., Gironda A., Llosa G., Mendoza E., Mitchell C., Mora C., Mora M., Padilla P., Piland R., Ponce C., Ramírez J. \& Varese M. 1999. Zona Reservada de Tambopata-Candamo. Conservación Internacional. Lima-Perú.

Barbarán G. 1998. Determinación de biomasa y carbono en los principales sistemas de uso del suelo en la zona de Campo Verde. Tesis profesional. Facultad de Ciencias Agropecuarias. Universidad Nacional de Ucayali. Pucallpa, Ucayali, Perú.

Brown K. 1992. Carbon Sequestration and Storage in Tropical Forests. Centre for Social and Economic Research on the Global Environment University of East Anglia. UK.

Brown S. \& Lugo A. 1992. Aboveground biomass estimates for tropical moist forests of the Brazilian Amazon. Interciencia. 17: 8-18.

Butcher P.N., Howard J.M., Regetz J.S., Semmens B.X. \& Vincent M.A. 1998. Evaluating the Carbon Sequestration Potential of Tropical Forests. Donald Bren School of Environmental Science and Management.

Chuvieco, E. 1995. Fundamentos de Teledetección espacial. Segunda Edición. Ediciones Rialp, Madrid.

Ciesla W.M. 1996. Cambio Climático, Bosques y Ordenación Forestal. Una Visión de Conjunto. FAO. Roma-Italia.

Concha J.Y., Alegre J.C. \& Pocomucha V. 2007. Determinación de las reservas de Carbono en la biomasa aérea de sistemas agroforestales de Theobroma cacao L. en el departamento de San Martín, Perú. Ecología Aplicada. 6(1,2): 75-82.

DeFries R.S., Houghton R.A., Hansen M.C., Field C.B., Skole D. \& Townshend J. 2002. Carbon emissions from tropical deforestation and regrowth based on satellite observations for the 1980s and 1990s. Proc. Natl Acad. Sci. USA. 99: 14256-14261.

Díaz R., Acosta M., Carrillo F., Buendía E., Flores E. \& Etchevers J.D. 2007. Determinación de ecuaciones alométricas para estimar biomasa y carbono en Pinus patula Schl. et Cham. Madera y Bosques. 13(1): 25-34.

Encarnación F. 1993. El bosque y las formaciones vegetales en la llanura amazónica del Perú. Alma Máter. 6: 95114.

Epstein P.R. \& Rogers C. 2004. Inside the Greenhouse. The impacts of $\mathrm{CO}_{2}$ and climate change on public health in the Inner City. The Center for Health and the Global Environment-Harvard Medical School, Boston-USA

Fearnside P.M. 1992. Forest biomass in Brazilian Amazonia: comments on the estimate by Brown and Lugo. Interciencia. 17: 19-27.

Fearnside P.M. 1994. Biomassa das florestas Amazônicas brasileiras. In Anais do Seminário Emissão X Seqüestro de CO2: pp. 95-124. Companhia Vale do Rio Doce (CVRD). Rio de Janeiro-Brasil.

Fearnside P.M. 1997. Greenhouse gases from deforestation in Brazilian Amazonia: Net committed emissions. Climatic Change. 35: 321-360.

Figueroa C., Etchevers J.D., Velázquez A. \& Acosta M. 2005. Concentración de carbono en diferentes tipos de vegetación de la Sierra Norte de Oaxaca. Terra. 23: 5764.

Freitas L., Otárola E., del Castillo D., Linares C., Martínez P. \& Malca D. 2006. Servicios ambientales de almacenamiento y secuestro de carbono del ecosistema aguajal en la Reserva Nacional Pacaya Samiria, LoretoPerú. Documento Técnico ${ }^{\circ}$ N29. IAAP. Domi nius Publicidad. Iquitos-Perú.

Gibbs H.K. 2006. Olson's major world ecosystem complexes ranked by carbon in live vegetation: an updated database using the GLC2000 land cover product.

(http://cdiac.ornl.gov/epubs/ndp/ndp017/ndp017b.html).

Gibbs H.K, Brown S., Niles J.O. \& Foley J.A. 2007. Monitoring and estimating tropical forest carbon stocks: making REDD a reality. Environ. Res. Lett. 2: 1-13.

Houghton R.A. 1999. The annual net flux of carbon to the atmosphere from changes in land use 1850-1990. Tellus B. 51: 298-313.

Husch B. 2001. Estimación del contenido de carbono de los bosques. Simposio Internacional Medición y Monitoreo de la Captura de Carbono en Ecosistemas Forestales, Valdivia, Chile.

IIAP. 2002. Estudio de Línea Base de secuestro de carbono en la cuenca del río Nanay. Iquitos-Perú.

INEI. 2010. Instituto Nacional de Estadística e Informática. (http://iinei.inei.gob.pe/iinei/SIRTOD/).

IPCC. 1996. Climate Change 1995 - Impacts, Adaptations and mitigation of climate change: scientific technical analysis. Contribution of Working Group II to the Second Assessment Report of the IPCC. Cambridge University Press. Cambridge-USA.

IPCC. 2000. Land use, Land use change, and forestry, a special report. Cambridge University Press, Cambridge, United Kingdome.

IPCC. 2001. Summary for policymakers. A report of working group I of the Intergovernamental Panel of Climate Change. (www.ipcc.ch).

IPCC. 2006. IPCC Guidelines for National Greenhouse Gas Inventories. Institute for Global Environmental Strategies, Japan.

Isagi Y., Kawahara T. \& Kamo K. 1993. Biomass and net production in a bamboo Phyllostachys bambusoides stand. Ecological Research. 8(2): 123-133.

Iverson L.R., Brown S., Grainger A., Prasad A. \& Liu D. 1993. Carbon sequestration in tropical Asia: an assessment of technically suitable forest lands using geographic information systems analysis. Climate Research. 3: 23-38.

Jandl R. 2001. Medición de tendencias en el tiempo del almacenamiento de carbono del suelo. Simposio Internacional Medición y Monitoreo de la Captura de Carbono en Ecosistemas Forestales, Valdivia, Chile.

Kerr S., Pfaff A.S.P. \& Sanchez A. 2001. The Dynamics of Deforestation and the Supply of Carbon Sequestration: Illustrative Results from Costa Rica. In Panayoutou T (ed) Central America Project, Environment: Conservation and Competitiveness: 409-431. Harvard University Press, Massachusetts, USA.

Kerr S., Lipper L., Pfaff A.S.P., Cavatassi R., Davis B., Hendy J. \& Sanchez A. 2004. Will Buying Tropical Forest Carbon Benefit The Poor? Evidence from Costa Rica. ESA Working paper 4-20. Agricultural and Development Economics Division. The Food and 
Agriculture Organization of the United Nations. RomeItaly.

Leith H. \& Whithacker R. 1975. Primary Productivity of the Biosphere. Ecological Studies v. 14. Springer-Verlag, New York-USA.

Márquez L. 2000. Elementos Técnicos para Inventarios de Carbono en uso del Suelo. Fundación Solar, Guatemala.

Nascimento H.E.M. \& Laurance W.F. 2002. Total aboveground biomass in central Amazonian rainforests: a landscape-scale study. Forest Ecology and Management. 168 (1-3): 311-321.

NETL. 2004. Developing the technology base and infrastructure to enable sequestration as a greenhouse gas mitigation option. National Energy Technology Laboratory. United States of America. (www.netl.doe.gov).

Phillips O.L., Malhi Y., Higuchi N., Laurance W.F., Nunes R.M., Vaxques R., Laurance L.V., Ferreira M., Stern M., Brown S. \& Grace J. 1998. Changes in the carbon balance of tropical forest: evidence from long-term plots. Science. 282: 439-442.

Pitman N.C.A. 2008. An overview of the Los Amigos watershed, Madre de Dios, southeastern Peru. (http://cicra.acca.org.pe).
Richards K.R. \& Stokes C. 2004. A review of forest carbon sequestration cost studies: a dozen years of research. Climatic Change 63: 1-48.

Raev I., Asan Ü. \& Grozev O. 1997. Accumulation of $\mathrm{CO}_{2}$ in the above-ground biomass of the forests In Bulgaria and Turkey in the recent decades. Proceedings of the XI World Forestry Congress 1: 131-138.

Rodríguez R., Jiménez J., Aguirre Ó.A. \& Treviño E.J. 2006. Estimación del Carbono almacenado en un bosque de niebla en Tamaulipas, México. Ciencia UANL. 9(2): 179-187.

Schlesinger W.H. \& Andrews J.A. 2000. Soil respiration and the global carbon cycle. Biogeochemistry. 48: 7-20.

Schulze E.D., Wirt Ch. \& Heimann M. 2000. Managing forest after Kyoto. Science. 289: 2058-2059.

Tipper R. 1998. Update on carbon offsets. Tropical Forest Update. 8(1): 2-5.

Torezan J.M.D. \& Silveira M. 2000. The biomass of bamboo (Guadua weberbaueri Pilger) in open forest of the southwestern Amazon. Ecotropica. 6(1): 71-76.

Winrock. 2006. Carbon Storage in the Los Amigos Conservation Concession, Madre de Dios, Perú. Winrock Intertational.

\footnotetext{
${ }^{1}$ Museo de Historia Natural Javier Prado-Universidad Nacional Mayor de San Marcos. Apartado 14-0434, Lima-Perú. cmartelg@gmail.com
} 